\title{
P3 Factor Influencing Endothelial Function in Type 2 Diabetes Mellitus with Newly Diagnosed Hypertension
}

\author{
Prachi Srivastava* \\ All India Institute of Medical Sciences, New Delhi, India
}

\section{ABSTRACT}

Background: Increased risk for cardiovascular diseases is associated with diabetes, can be partially attributed to endothelial dysfunction. However, the cumulative factors leading to endothelial dysfunction in Type 2 DM are not clear. In the current study, a novel attempt was made to understand factors influencing endothelial function (EF) in humans using Angiotensin Converting Enzyme (ACE) inhibition as a tool to alter EF.

Methods: Brachial Flow-mediated-dilation (FMD) was measured using ultrasonography in 60 diabetics with newly-diagnosed hypertension. Serum E-selectin, Vascular-Cell-Adhesion-Molecule-1 (VCAM-1), hsCRP, IL-10, Renin, Ang 1-7, Ang II and ACE 2 were measured using ELISA. Amplitude of change, calculated as absolute difference in values obtained at baseline (V1) from those after 3 months (V3) (V3-V1), of biomarkers were correlated with change in FMD and E-selectin after 3 months of ACE inhibition.

Results: FMDV3-V1 showed significant negative correlation with hsCRPV3-V1, FSV3-V1, PPSV3-V1 and HbA1cV3-V1 $[(r=-0.3, p=0.02),(r=-0.31, p=0.01),(r=-0.27, p=0.03)(r=-0.3, p=0.02)$, respectively $]$ and significant positive correlation with Ang1-7V3-V1 $(r=0.39, p=0.002)$. E-selectin correlated positively with FSV3-V1, PPSV3-V1, HbA1cV3-V1, VCAM-1V3-V1 and ReninV3-V1 [ $(r=0.35, p=0.006),(r=0.32, p=0.01),(r=0.45, p=0.0005),(r=0.66, p<0.001),(r=0.53$, $p<0.0001)$ respectively].

Conclusion: A balance between the Renin/ Ang II and Ang 1-7/ACE2 axis plays a significant role in EF. Additionally, reduction in blood sugar levels and vascular inflammation also improves EF.

\section{REFERENCES}

[1] De Vriese AS, Verbeuren TJ, Van de Voorde J, Lameire NH, Vanhoutte PM. Endothelial dysfunction in diabetes. Br J Pharmacol 2000;130:963-74.

[2] Bernardi S, Michelli A, Zuolo G, Candido R, Fabris B. Update on RAAS modulation for the treatment of diabetic cardiovascular disease. J Diabetes Res 2016;2016:8917578.

[3] Srivastava P, Badhwar S, Chandran DS, Jaryal AK, Jyotsna VP, Deepak KK. Imbalance between Angiotensin II - Angiotensin (1-7) system is associated with vascular endothelial dysfunction and inflammation in type 2 diabetes with newly diagnosed hypertension. Diabetes Metab Syndr 2019;13:2061-8.

(c) 2019 Association for Research into Arterial Structure and Physiology. Publishing services by Atlantis Press International B.V. This is an open access article distributed under the CC BY-NC 4.0 license (http://creativecommons.org/licenses/by-nc/4.0/). 